\title{
BMJ Open Oral propranolol for prevention of threshold retinopathy of prematurity (ROPROP): protocol of a randomised controlled trial
}

\author{
Christoph Bührer, ${ }^{1}$ Ömer Erdeve, ${ }^{2}$ Dirk Bassler, ${ }^{3}$ Benjamin Bar-Oz ${ }^{4}$
}

To cite: Bührer C, Erdeve 0̈, Bassler D, et al. Oral propranolol for prevention of threshold retinopathy of prematurity (ROPROP): protocol of a randomised controlled trial. BMJ Open 2018;8:e021749. doi:10.1136/ bmjopen-2018-021749

- Prepublication history for this paper is available online. To view these files, please visit the journal online (http://dx.doi. org/10.1136/bmjopen-2018021749).

Received 15 January 2018

Revised 20 April 2018

Accepted 5 June 2018
Check for updates

${ }^{1}$ Department of Neonatology, Charité Universitätsmedizin Berlin, Berlin, Germany ${ }^{2}$ Division of Neonatology, Ankara University School of Medicine Children's Hospital, Ankara, Turkey

${ }^{3}$ Department of Neonatology, Universitätsspital Zürich, Zurich, Switzerland

${ }^{4}$ Department of Neonatology, Hadassah Medical Center, Jerusalem, Israel

Correspondence to Dr Christoph Bührer; christoph.buehrer@charite.de

\section{ABSTRACT}

Introduction Retinopathy of prematurity (ROP) is a disease observed in extremely premature infants characterised by visioning-threatening retinal vessel proliferation. Propranolol, a drug used for decades in newborn infants with heart diseases, hypertension and thyrotoxicosis and licenced for infantile haemangiomas, may be effective in halting progression of ROP to severe stages, as suggested by preliminary data from small studies.

Methods and analysis ROPROP is an investigatorinitiated, multicentre, placebo-controlled double-blind, randomised controlled trial aiming to assess the safety and efficacy of orally administered propranolol to reduce the risk of threshold ROP (stage 3) in extremely preterm infants at 48 weeks postmenstrual age (primary objective) and the rate of infants requiring local interventions for severe ROP (secondary objective). Key inclusion criteria: gestational age $<28$ weeks, birth weight $<1250 \mathrm{~g}$, postmenstrual age $\geq 31$ and $<37$ weeks, incipient ROP (stage 1 or 2, with or without plus disease) and written informed consent by parents or legal guardian. Key exclusion criteria: requirement for open-label propranolol treatment, major congenital malformations (including those with cerebrovascular malformations), known chromosomal anomalies, colobomas and other eye malformations, atrioventricular block grade 2 or 3 and comedication with antiarrhythmics, clonidine, insulin (pharmacodynamic interaction), phenobarbital or rifampicin (pharmacokinetic interaction). The intervention consists of oral propranololhydrochloride $(1.6 \mathrm{mg} / \mathrm{kg} /$ day in three to four divided dosages) or placebo until discharge, for a maximum of 10 weeks. Analysis is by intention to treat.

Ethics and dissemination The protocol has received ethical and regulatory approval. Results will be published after peer review irrespective of the study outcome. Trial registration numbers NCT03083431, EudraCT\# 2017-002124-24 (EUCTR), 00013730 (DRKS); Pre-results.

\section{INTRODUCTION}

Propranolol is a non-selective $\beta$ blocker, first discovered in $1964^{1}$ by James Whyte Black (Nobel Prize 1988). It is on the WHO's List of Essential Medicines for Children ${ }^{2}$ and worldwide available as a generic medication without
Strengths and limitations of this study

- Repurposing of a well-known drug for a rare disease.

- Large, multicentre trial addressing a major public health concern.

- No commercial interests (investigator initiated and funded by public agencies).

No central review of outcome possible.

age restrictions. Its long-standing indications include treatment of high blood pressure, a number of types of irregular heart rate, thyrotoxicosis, migraine prophylaxis, performance anxiety and essential tremor. In infants and children, propranolol is used for prevention of paroxysmal supraventricular tachycardia, prevention of sudden ventricular fibrillation in long QT syndrome, prevention of blue spells in tetralogy of Fallot and treatment of hypertension in children with renal or cardiovascular disease. Moreover, propranolol has been found to lead to the regression of infantile haemangiomas and has been licenced in 2014 for this purpose in the European Union, Switzerland and the USA.

Retinopathy of prematurity (ROP) is a disorder of the developing retina observed only in low birthweight preterm infants that may lead to blindness in a small but significant percentage of those infants. In preterm infants, retinal vessels are incompletely developed at birth and display a growth spurt peaking around 4-8 weeks before due term. Ophthalmoscopies performed during this growth spurt may reveal abnormal proliferation of blood vessels that recede once vascularisation is complete. If the neovascularisation, however, progresses to extraretinal fibrovascular proliferation into the vitreous (threshold ROP), there is a high risk of retinal detachment with subsequent blindness. Threshold ROP is associated with polymorphisms in a variety of genes, none of 
which cause retinopathy in the absence of prematurity. Vision-threatening ROP affects $10 \%-15 \%$ of extremely preterm infants with a gestational age below 28 weeks ( $0.7 \%$ of all newborns), with an overall incidence of about 5 cases per 1000000 inhabitants (orphan disease).

Currently available treatment of threshold ROP, consisting of laser ablation of the peripheral retina or intravitreal injection of antagonists of vascular endothelial growth factor (VEGF), may prevent most but not all cases of permanent ROP-mediated blindness. Treated ROP is a strong independent predictor of later neurodevelopmental impairment in childhood and adolescence. ${ }^{3-5}$ Both types of local treatment are associated with significant costs and side effects. ${ }^{6}$ Ablative laser surgery leads to permanent destruction of the peripheral retina (scars), while normal-appearing peripheral retinal vessels have been found to continue after intravitreal treatment. A large randomised controlled trial (RCT) has shown superior short-term and long-term results for intravitreal injections of the anti-VEGF antibody, bevacizumab, as compared with laser treatment. ${ }^{78}$ Intravitreal bevacizumab treatment, however, is completely off-label, as the antibody is not manufactured for intravitreal use and has never been licenced for infants. Other anti-VEGF antagonists made for intravitreal administration (ranibizumab, pegabtanib and aflibercept) are also not licenced in infants and have never been tested in an RCT. Despite these limitations, intravitreal anti-VEGF administration has become the preferred treatment for threshold ROP in several countries. ROP prevention and treatment figure high on the list of priority conditions requiring study in newborns.

The pathophysiological pattern of ROP is reminiscent to the natural history of infantile haemangiomas, which are also more common in preterm infants. There is conflicting evidence as to the antiangiogenic efficacy of propranolol in mouse models of oxygen-induced retinopathy that recapitulate several aspects of ROP ${ }^{9-11}$ Preliminary results from two open-label comparison studies ${ }^{12} 13$ and three RCTs ${ }^{14-16}$ suggest that oral propranolol administered at $1-2 \mathrm{mg} / \mathrm{kg} /$ day may be effective in halting progression of ROP to severe stages. In an open-label comparison study ${ }^{12}$ conducted in Chile, 2 of 20 preterm infants with ROP $\geq$ stage 2 on propranolol $(1.5 \mathrm{mg} / \mathrm{kg}$ / day in three divided doses) underwent laser treatment, as opposed to 13 of 27 historical control infants $(p<0.01)$. In an open-label single-centre comparison study from Kayseri, Turkey, ${ }^{13} 4$ of 83 preterm infants on propranolol $(2 \mathrm{mg} / \mathrm{kg} /$ day in four divided doses $)$ underwent ablative laser surgery for ROP, as opposed to 8 of 88 control infants $(\mathrm{p}>0.1)$. Two RCTs (NCT01238471 and NCT0107915) allocated a total of 72 preterm infants with stage 2 ROP to oral propranolol or control ${ }^{1415}$ and found a similar reduction of infants requiring treatment (relative risks 0.44 and 0.42 , respectively). The relative risk of the two trials combined is 0.44 (95\% CI 0.19 to 1.02 ), translating in a number needed to treat of $5 .{ }^{17} \mathrm{~A}$ third RCT (CTRI/2013/11/004131) randomised 109 preterm infants with gestational age between 26 and 32 weeks to prophylactic propranolol $(1 \mathrm{mg} / \mathrm{kg} /$ day divided in two doses) on the day 7 of life or placebo, until a corrected gestational age of 37 weeks or complete vascularisation of retina, whichever was later. ${ }^{16}$ Of 51 evaluable infants on propranolol, 13 infants underwent treatment for ROP (laser or intravitreal VEGF antagonist), as opposed to $24 / 51$ controls $(\mathrm{p}=0.0235)$.

An overview of published results of trials is given in table 1.

Further registered clinical trials evaluating the use of oral propranolol in preterm infants with early stages of

Table 1 Overview of pilot trials assessing oral propranolol for treatment or prevention of retinopathy of prematurity (ROP)

\begin{tabular}{|c|c|c|c|c|c|c|c|}
\hline Study & $\begin{array}{l}\text { Participants } \\
\text { propranolol } \\
\text { /controls }\end{array}$ & $\begin{array}{l}\text { Gestational } \\
\text { age (weeks) }\end{array}$ & $\begin{array}{l}\text { Birth } \\
\text { weight (g) }\end{array}$ & $\begin{array}{l}\text { Dosage } \\
\text { propranolol } \\
\text { (mg/kg/day) }\end{array}$ & Duration (day) & $\begin{array}{l}\text { Rate ROP } \\
\text { intervention } \\
\text { propranolol } \\
\text { versus controls }\end{array}$ & Side effects \\
\hline Filippi et $a l^{15}$ & $26 / 26$ & $\begin{array}{l}23-25 \\
26-32\end{array}$ & $\begin{array}{l}691 \pm 127 \\
860 \pm 244\end{array}$ & $\begin{array}{l}1.0(4 \times 0.25) \\
2.0(4 \times 0.5)\end{array}$ & $66 \pm 31(6-90)$ & $\begin{array}{l}4 / 25 \text { versus } \\
10 / 26\end{array}$ & $\begin{array}{l}5 / 26 \\
\text { hypotension } \\
\text { bradycardia } \\
\text { bronchospasm }\end{array}$ \\
\hline Sanghvi et $a l^{16}$ & $55 / 54$ & $26-32$ & $1235 \pm 280$ & $1.0(2 \times 0.5)$ & $\begin{array}{l}32 \\
(7-72)\end{array}$ & $\begin{array}{l}13 / 51 \text { versus } \\
24 / 51\end{array}$ & None \\
\hline Bancalari et $a l^{12}$ & $20 / 27$ & $26.6 \pm 1.6$ & $937 \pm 285$ & $1.5(3 \times 0.5)$ & $58 \pm 18$ & $\begin{array}{l}2 / 20 \text { versus } \\
14 / 27\end{array}$ & None \\
\hline
\end{tabular}


ROP (WHO International Clinical Trials Registry Platform, http://apps.who.int/trialsearch/default.aspx) have no results yet:

- IRCT2015102524681N1 is a parallel-assignment non-placebo-controlled trial from Guilen, Iran, for infants with early stages of ROP using oral propranolol at $2 \mathrm{mg} / \mathrm{kg} /$ day divided in four doses for infants with a gestational age of 26-31 weeks and $1 \mathrm{mg} / \mathrm{kg} /$ day divided in four doses for infants with a gestational age of 23-25 weeks (target sample size, $\mathrm{n}=50$ ). Recruitment started in 2015 and is ongoing.

- NCT02977000 is a single-group assignment trial from Zhengzhou, China, which treats preterm newborns with a gestational age below 32 weeks and stage 2 ROP with oral propranolol (target sample size, $\mathrm{n}=100$ ). Recruitment started in 2016 and is ongoing.

- NCT03038295 is placebo-controlled RCT from Guangdong, China, comparing oral propranolol $(0.25 \mathrm{mg} / \mathrm{kg} /$ day $)$ and propranolol eye drops $(0.2 \%)$ versus placebo in preterm infants below $1500 \mathrm{~g}$ birth weight and early stages of ROP (target sample size, $\mathrm{n}=100$ ). The study is not recruiting yet.

An Italian trial with topical therapy using $0.1 \%$ propranolol eye drops (EudraCT 2013-002062-39) planned according to the Simon optimal two-stage design for phase II clinical trials was discontinued early after recruiting 23 patients before starting the second stage since the number of failures was above the set threshold. ${ }^{18}$ Subsequently, a multicentre open-label pilot study (NCT02504944, EudraCT 2014-0 05 472-29) investigating the use of 0.2\% propranolol eye drops in infants with stage 1 ROP (target sample size, $n=96$ ) has been launched that is currently recruiting patients. ${ }^{19}$

Based on the effect size calculated from the trials that have results, all of these individual trials are considered underpowered to detect a significant difference in major ophthalmic outcomes. The ROPROP trial has been designed to investigate the safety and efficacy of oral propranolol administered to preterm infants with incipient ROP aimed at reducing the rate of progression to severe ROP with an adequate sample size.

\section{METHODS AND ANALYSIS \\ Patients}

Each patient must meet all of the following criteria to be enrolled in this double-blind RCT:

- Preterm infant born before 28 weeks' gestation.

- Birth weight below $1250 \mathrm{~g}$.

- Alive at 5 weeks of age.

- Postmenstrual age $31^{0} /{ }_{7}-36 \% /{ }_{7}$ weeks.

- Ophthalmoscopic evidence of incipient ROP (stage 1 or 2 , with or without plus disease).

- Written informed consent by parents or legal guardian, including saving and propagation of pseudonymous medical data for study purposes.

Patients meeting any of the following criteria are not eligible for enrolment:
- ROP stage 3 at time of inclusion (endpoint already reached).

- Thyrotoxicosis, arterial hypertension or congenital heart diseases requiring open-label propranolol treatment (such as tetralogy of Fallot, paroxysmal supraventricular tachycardia or long QT syndrome).

- Atrioventricular block grade 2 or 3 (contraindication for propranolol).

- Sinoatrial block (contraindication for propranolol).

- Uncontrolled heart failure or cardiogenic shock (contraindication for propranolol).

- Acute severe infection (inclusion may be postponed until infection has resolved).

- Bronchial asthma.

- Major congenital malformations or known chromosomal anomalies.

- Colobomas and other eye malformations.

- PHACE syndrome (posterior fossa anomalies; large infantile haemangiomas of the face, neck and/or scalp; arterial lesions; cardiac abnormalities/coarctation of the aorta; and eye anomalies) (risk of cerebrovascular complications).

- Very large haemangioma (risk of hyperkalaemia), as judged by the attending physician.

- Heart rate consistently ( $>1$ hour) $<100 / \mathrm{min}$.

- Non-invasive mean arterial pressure consistently ( $>1$ hour) $<40 \mathrm{~mm} \mathrm{Hg}$.

- Medication of the infant or the mother if breast feeding with clonidine, reserpine, angiotensin converting enzyme (ACE) inhibitors, angiotensin-receptor antagonists (contraindicated in preterm infants) or antiarrhythmic drugs including amiodarone, propafenone, lidocaine, digoxin/digitoxin, quinidine, verapamil, diltiazem and bepridil (pharmacodynamic interaction).

- Medication of the infant with rifampicin or phenobarbitone (enhanced metabolic clearance).

- Concurrent treatment with insulin (risk of hypoglycaemia).

- Severe liver dysfunction (GPT, alanine aminotransferase $>900 \mathrm{U} / \mathrm{L})$.

- Chronic kidney impairment (serum creatinine $>1.3 \mathrm{mg} / \mathrm{dL}(100 \mu \mathrm{M}))$

- Persistent hypoglycaemia (blood glucose $<36 \mathrm{mg} / \mathrm{dL}$ $(2.0 \mathrm{mM})$ in three consecutive samples immediately preceding enrolment).

- Persistent hyperkalaemia (venous serum potassium $>5.9 \mathrm{mM}$ in three consecutive samples immediately preceding enrolment).

- Persistent neutropenia (absolute neutrophil counts $<1000 / \mu \mathrm{L}$ in three consecutive samples immediately preceding enrolment).

- Known hypersensitivity to propranolol or any of the excipients.

- Prinzmetal's angina, Raynaud's phenomenon (severe peripheral arterial circulatory disturbance) or pheochromocytoma (contraindications for propranolol in adults, not occurring in newborn infants). 
- Participating in another pharmacological interventional clinical trial.

- Any circumstances that make the investigator believe that participation in the study leads to exceptional medical or organisational problems for the patient.

\section{Enrolment, randomization and allocation concealment}

Infants who meet all inclusion criteria and whose parents/ legal representatives have given their written informed consent will be reported to the trial centre and are the subjects of the central enrolment procedure. Each participating unit will keep an enrolment log of preterm infants born before 28 weeks' gestation with a birth weight below $1250 \mathrm{~g}$, containing date of birth, sex, gestational age, birth weight, death/date of death, transfer/dates of transfer, discharge/date of discharge, dates and results of ophthalmoscopies (International Committee for the Classification of Retinopathy of Prematurity (ICROP) stage).

Every patient will receive an enrolment number, which will be unique for this individual patient. Patients who pass all inclusion and exclusion criteria, laboratory tests and ECG and are therefore eligible for the study will be randomised. In order to distribute possible confounding variables evenly in both groups, the patients will be randomised, with stratification of centre and gestational age. This randomisation will be done centrally using the randomisation tool within the study software secuTrial. The randomisation scheme incorporating variance minimisation will be used to assign infants, in a 1:1 ratio, to verum or placebo, with stratification according to gestational age ( $<26$ weeks vs $\geq 26$ weeks) and study site. Sequences of patient numbers will be assigned to each study centre and kept confidential, using sequentially numbered blinded medication boxes. The study medication (propranolol or placebo) will be randomly assigned to each patient number. Each bottle of the study medication has a unique medication number in the study medication list (unblinded) and will be manually linked to the assigned random patient number. The randomisation documentation ('key list') is subject to monitoring. Treatment allocation will only be known to the pharmacist and the unblinded person. Neither the neonatologists taking care of the baby, nor the ophthalmologists performing the opthalmoscopies (the actual outcome assessors) will be aware of the assignment.

\section{Intervention and comparator}

The intervention consists of oral propranolol $(1.6 \mathrm{mg}$ propranolol hydrochloride/kg/day in 3-4 divided dosages) given for 4-10 weeks (depending on postmenstrual gestational age at birth) or placebo. The study medication $(1 \mathrm{mg} / \mathrm{mL}$ propranolol hydrochloride solution or placebo) will be provided and labelled by the good manufacturing practice (GMP) licenced University Pharmacy of Erlangen, Germany, as a solution in screw-capped bottles. The oral solution contains sucrose $(25 \mathrm{~g} / 100 \mathrm{~mL})$, potassium sorbate $(0.15 \mathrm{~g} / 100 \mathrm{~mL})$, citric acid monohydrate $(0.07 \mathrm{~g} / 100 \mathrm{~mL})$, orange flavour and distilled water as excipients. The bottles are stored at room temperature. The solution is drawn into a syringe and administered by mouth or gastric gavage together with feeds. If the infant does not drink the medication, it will be given by gavage, but there is no replacement dose in case an infant vomits. If enteral feeding is stopped completely for any reason, the study medication will be temporarily discontinued and gradually resumed when enteral feeding is reinstituted.

The starting dose is $0.4 \mathrm{mg} / \mathrm{kg} /$ day $(=0.4 \mathrm{~mL} / \mathrm{kg} / \mathrm{d})$ by mouth or gavage in four divided dosages (ie, every $6 \pm 1.5$ hours) together with feeds, followed by incremental increases by $0.4 \mathrm{mg} / \mathrm{kg}$ every other day to a final dose of $1.6 \mathrm{mg} / \mathrm{kg} /$ day (concentrations pertain to propranolol hydrochloride, to convert into propranolol base, multiply by 0.878 ). Once an infant is switched to feeding every 4 hours, the total dose may be divided in three divided dosages (ie, every $8 \pm 2$ hours). The duration of the study medication treatment is limited to 10 weeks. The study drug is reduced by $50 \%$ and discontinued the following day prior to discharge, when a decision has been made for local ROP treatment or when the infants is scheduled for surgery (eg, for inguinal hernia repair). No study medication will be given to patients out of hospital.

The study medication will be discontinued temporarily in the event of a lower respiratory infection associated with dyspnoea and wheezing or for any adverse clinical symptoms, as decided by the attending physicians. As soon as symptoms resolve, the maintenance dose is to be resumed but reduced by $50 \%$ and readjusted to the original dose after 3 days. Attending physicians can hold or decrease doses of study drugs at their discretion in case of worsening disease or recurring adverse clinical symptoms. The study medication will be permanently discontinued in the event of isolated bronchospasm or severe liver disease.

The following drugs may not be given concomitant with the study medication: antiarrhythmic drugs (all classes including amiodarone, propafenone, lidocaine, digoxin/digitoxin and calcium channel blockers), clonidine, reserpine, ACE inhibitors/angiotensin receptor antagonists (contraindicated in preterm infants), inhalational anaesthetics (except during emergencies), rifampicin and phenobarbitone.

If an infant requires open-label propranolol during the study, the infant will be taken off the study drug, and the data will be censored for the efficacy analysis.

If a mother who breast feeds her infant starts taking propranolol, steroids, rifampicin or phenobarbitone, the investigator will be informed while breast feeding may continue. It will be recorded, but this does not imply discontinuation of the study and will not change the primary or secondary efficacy analysis which is by intention to treat.

If a mother who breast feeds her infants starts to take antiarrhythmic drugs (all classes including amiodarone, propafenone, lidocaine, digoxin/digitoxin and calcium channel blockers), clonidine, reserpine, ACE inhibitors 
or angiotensin-receptor antagonists, the infant will be taken off the study drug, and the data will be censored for the efficacy analysis.

\section{Outcome and time frame}

The primary endpoint for efficacy is survival until 48 weeks postmenstrual age without ROP stage 3 (any zone), as diagnosed according to the International Committee for the Classification of Retinopathy of Prematurity Revisited. ${ }^{20}$ ROP stage 3 is characterised by extraretinal proliferation of blood vessels into the vitreous and a threshold for local therapy (laser or intravitreal injections of VEGF antagonists). Because there may be more than one ROP stage in the same infant (and actually the same eye), the ROP stage of the infant in this trial is determined by the most severe manifestation present. Retinopathy is diagnosed by an ophthalmologist blinded to the group assignment (experimental intervention or control). Death is included as a competing terminal event as it may occur before ROP has progressed to its peak stage.

The secondary endpoint for efficacy is survival until 48 weeks postmenstrual age without local treatment for ROP (ablative laser surgery or intravitreal injections of anti-VEGF agents). Treated ROP is associated with neurodevelopmental impairment at 2, 5 and 11 years of age. ${ }^{3-5}$

Safety is gauged by the incidence of the following major events: death, necrotising enterocolitis (requiring surgery), culture-proven sepsis or meningitis (defined as growth of a recognised pathogen not counting coagulase-negative staphylococci in blood or cerebrospinal fluid in an infant treated for at least 5 days with intravenous antibiotics and a rise of $\mathrm{C}$ reactive protein to more than $10 \mathrm{mg} / \mathrm{L}$ during the first 72 hours of antibiotic treatment), symptomatic hypoglycaemia (blood glucose $<30 \mathrm{mg}$ / $\mathrm{dL}$ requiring intravenous glucose administration for 48 hours or more) and emergency endotracheal intubation (excluding elective intubation for surgery).

Exploratory continuous scale secondary outcomes are duration of supplemental oxygen and duration of hospitalisation (relative to the date of due term delivery), lowest heart rate and mean arterial pressure while the infant received study medication, average relative gain of weight and head circumference between start and end of the study medication.

\section{Sample size calculation}

The ROPROP study is designed to demonstrate the superiority of oral propranolol in comparison with placebo in increasing the rate of infants surviving to 48 weeks postmenstrual age without threshold ROP (primary endpoint). Assuming a rate of $30 \%$ of infants who will progress to threshold ROP or die, ${ }^{21} 262$ infants (131 in each group) will have to be analysed to have $80 \%$ power (at a two-sided alpha level of $5 \%$ ) to detect a $50 \%$ lower risk in the treatment group (two-sided Fisher's exact test-based calculations, $\mathrm{G}^{*}$ power 3.1.9.2, 2014). ${ }^{22}$ With an anticipated loss to follow-up of $5 \%$ (withdrawal or other reasons), the total number of infants allocated to treatment will be 276. Assuming that $10 \%$ of eligible patients will not be recruited for lack of parental consent and $3 \%$ for medical or organisational exclusion criteria, a total of 312 patients will be assessed for eligibility. This translates to 624 patients screened by funduscopy, assuming a rate of incipient ROP of $50 \%$, and 718 infants below 28 weeks' gestational age admitted to neonatal care, assuming an early mortality rate of $15 \%$.

\section{Statistical analysis plan}

The ROPROP study is designed to demonstrate the superiority of oral propranolol in comparison to placebo in increasing the rate of infants surviving to 48 weeks postmenstrual age without ROP stage 3 or more (primary endpoint).

\section{Null hypothesis}

There is no difference in the rate of infants surviving to 48 weeks postmenstrual age without ROP stage 3 or more between infants treated with oral propranolol and infants treated with placebo.

\section{Alternative hypothesis}

There is a difference in the rate of infants surviving to 48 weeks postmenstrual age without ROP stage 3 or more between infants treated with oral propranolol and infants treated with placebo.

The following populations will be described and analysed:

- The screened population will comprise all screened patients. The screened population will be described in terms of the reasons for exclusion from randomisation. No analysis will be done for the screened population.

- The intention-to-treat (ITT) population includes all randomised patients who received at least one dose of the study medication, with the exception of patients who are randomised in contradiction to one of the inclusion or exclusion criteria.

- The 'Per Protocol population' (PPP) will be based on the ITT. Patients treated with open-label oral propranolol will be excluded from the PPP.

- The safety population will include all infants who ever received the study drug.

The primary and secondary endpoints will be analysed in the ITT and PPP, while safety endpoints will be analysed in the safety population.

The primary endpoint for efficacy is survival until 48 weeks postmenstrual age without ROP stage 3 or more (any zone), as diagnosed according to the ICROP Revisited (12). The proportion of infants who have reached the primary endpoint will be compared and statistically assessed by means of a two-sided Fisher's exact test to examine the null hypothesis of equal proportions in the groups of infants allocated to placebo and to oral propranolol, respectively. Effect sizes will be described by calculating the relative risk as well as the OR and 95\% CIs. 
The confirmatory analysis of the primary efficacy endpoint will be by ITT, based on all patients who were randomised according to the inclusion and exclusion criteria and who received at least one dose of the study medication.

To consider the influence of site and gestational age ( $<26$ weeks vs $\geq 26$ weeks), these variables will be included in a logistic regression analysis. Survival until week 48 without ROP stage 3 or more will be the dependent variable while treatment group, site and gestational age will be the independent variables.

To handle patients with unknown status of the primary endpoint, a sensitivity analysis will be carried out in three steps: (1) patients with missing values excluded (confirmatory analysis); (2) patients with missing values included, primary endpoint 'yes' (sensitivity analysis); and (3) patients with missing values, primary endpoint 'no' (sensitivity analysis) (best and worst case scenario).

The secondary endpoint for efficacy is survival until 48 weeks postmenstrual age without local treatment for ROP (ablative laser therapy or intravitreal injections of anti-VEGF agents). It will be analysed analogously to the primary endpoint.

The parameters for assessing safety are rates of death prior to discharge, necrotising enterocolitis (requiring surgery), culture-proven sepsis or meningitis (defined as growth of a recognised pathogen not counting coagulase-negative staphylococci in blood or cerebrospinal fluid in an infant treated at least 5 days with intravenous antibiotics and a rise of $\mathrm{C}$ reactive protein to more than $10 \mathrm{mg} / \mathrm{L}$ during the first 72 hours of antibiotic treatment), symptomatic hypoglycaemia (blood glucose $<30 \mathrm{mg} / \mathrm{dL}$ requiring intravenous glucose administration) and emergency endotracheal intubation (excluding intubation for surgery).

Continuous scale safety outcomes are duration of supplemental oxygen and duration of hospitalisation (relative to the date of due term delivery), lowest heart rate and mean arterial pressure while the infant received study medication, average weekly gain of weight and head circumference between start and end of the study medication.

Summary tables will list discrete variables by absolute and relative frequencies, and continuous variables will be given by median, minimum, maximum and $\mathrm{IQR}$ for each treatment group. Discrete variables will be compared using Fisher's exact tests, while continuous variables will be compared by the Wilcoxon test.

Adverse events (AE) and serious adverse events (SAE) will be analysed descriptively per group using absolute and relative frequencies.

The significance level in all tests will be set at 0.05 , except for the interim analysis. All $\mathrm{p}$ values except for the confirmatory analysis and the interim analysis of the primary endpoint are considered to be exploratory (no adjustment for multiple testing).

One formal interim analysis for efficacy is planned, after half of the patients have been enrolled in the trial and have reached 48 weeks' gestational age. The statistical observation will be done for a significance level of 0.001 (two sided) (adjustment according to Peto-Haybittle). The interim analyses will be conducted by a statistician using coded group labels A and B blinded for the actual therapy groups.

To allow for patient-level meta-analyses, deidentified individual participant data that underlie the results reported will be supplied to investigators whose proposed use of the data has been approved by an independent review committee (learnt intermediary) during a limited period of time for individual participant data meta-analysis. For strict deidentification, only year of birth (instead of date of birth), country (but not site) and birth weight category (by $100 \mathrm{~g}$ intervals) will be allowed.

\section{Patient and public involvement}

Patients or public were not involved in the design or conduct of the study, and no attempt was made to assess the burden of the intervention by patients themselves.

\section{ETHICS AND DISSEMINATION}

Ethical and regulatory approval will be obtained for each participating site prior to commencement of recruitment in all countries (Germany, Israel, Switzerland and Turkey). The trial has not started recruitment at the time the protocol is being submitted for publication. The results of the trial will be published irrespective of the outcome of the study.

Acknowledgements The authors would like to thank Slavica Dimitrovska for excellent administrative support to prepare the conduct of the trial. Thanks also to the further members of the coordinating committee, Claudia Knöpfli, Emel Okulu, Ilanit Linzer, Şerife Suna Oğuz, Christoph Rüegger, Matthias Fröhlich, Matthias Roth-Kleiner, Merih Çetinkaya and Thomas Schmitz, for helpful advice, and to Ralph Heimke-Brinck and Tobias Borst for preparation of the study medication and revising the investigators' brochure. We also acknowledge support from the German Research Foundation (DFG) and the Open Access Publication Fund of Charité Universitätsmedizin Berlin.

Collaborators Slavica Dimitrovska; Claudia Knöpfli; Emel Okulu; Ilanit Linzer; Şerife Suna 0ğuz; Christoph Rüegger; Matthias Fröhlich; Matthias Roth-Kleiner; Merih Çetinkaya; Thomas Schmitz; Ralph Heimke-Brinck; Tobias Borst.

Contributors The authors who have embarked previously on collaborative research concerning the diagnosis and treatment of ROP jointly drafted the E-Rare-3 proposal. All authors receive funding by the national agencies, act as principle investigators for their own countries and jointly form the steering committee. The protocol was drafted by $\mathrm{CB}$ and critically revised by ÖE, DB and BB-0.

Funding The trial is funded within the framework of the E-Rare-3 call 2016 (clinical research for new therapeutic uses of already existing molecules (repurposing) in rare diseases) by the German Federal Ministry of Education and Research (BMBF, grant \#01GM1703), the Swiss National Science Foundation (SNF, grant \#32ER30-173677), the Scientific and Technological Research Council of Turkey (TÜBITAK, grant \#117S537) and the Israeli Ministry of Health (MOH, grant \#3-0000-13836 ERANET-RARE-3).

Disclaimer The funders have no role in the analysis of the data, writing of the report, or the decision to submit the report for publication.

Competing interests None declared.

Patient consent Not required.

Ethics approval Ethikkommission Landesamt für Gesundheit und Soziales Berlin. 
Provenance and peer review Not commissioned; externally peer reviewed.

Open access This is an open access article distributed in accordance with the Creative Commons Attribution Non Commercial (CC BY-NC 4.0) license, which permits others to distribute, remix, adapt, build upon this work non-commercially, and license their derivative works on different terms, provided the original work is properly cited and the use is non-commercial. See: http://creativecommons.org/ licenses/by-nc/4.0/

(C) Article author(s) (or their employer(s) unless otherwise stated in the text of the article) 2018. All rights reserved. No commercial use is permitted unless otherwise expressly granted.

\section{REFERENCES}

1. Black JW, Crowther AF, Shanks RG, et al. A new adrenergic betareceptor antagonist. Lancet 1964:1:1080-1.

2. WHO. WHO expert committee on selection and use of essential medicines. WHO model list of essential medicines for children (6th edition). 2017 http://www.who.int/medicines/publications/ essentialmedicines/6th_EMLc2017.pdf2017

3. Bassler D, Stoll BJ, Schmidt B, et al. Using a count of neonatal morbidities to predict poor outcome in extremely low birth weight infants: added role of neonatal infection. Pediatrics 2009;123:313-8.

4. Schmidt B, Roberts RS, Davis PG, et al. Prediction of late death or disability at age 5 years using a count of 3 neonatal morbidities in very low birth weight infants. J Pediatr 2015;167:982-6.

5. Farooqi A, Hägglöf B, Sedin G, et al. Impact at age 11 years of major neonatal morbidities in children born extremely preterm. Pediatrics 2011;127:e1247-57.

6. Mueller B, Salchow DJ, Waffenschmidt E, et al. Treatment of type I ROP with intravitreal bevacizumab or laser photocoagulation according to retinal zone. $\mathrm{Br} J$ Ophthalmol 2017;101:365-70.

7. Mintz-Hittner HA, Kennedy KA, Chuang AZ, et al. Efficacy of intravitreal bevacizumab for stage $3+$ retinopathy of prematurity. $N$ Engl J Med 2011;364:603-15.

8. Geloneck MM, Chuang AZ, Clark WL, et al. Refractive outcomes following bevacizumab monotherapy compared with conventional laser treatment: a randomized clinical trial. JAMA Ophthalmol 2014;132:1327-33.
9. Martini $\mathrm{D}$, Monte MD, Ristori $\mathrm{C}$, et al. Antiangiogenic effects of $\beta 2$ -adrenergic receptor blockade in a mouse model of oxygen-induced retinopathy. J Neurochem 2011;119:1317-29.

10. Ristori $C$, Filippi $L$, Dal Monte $M$, et al. Role of the adrenergic system in a mouse model of oxygen-induced retinopathy: antiangiogenic effects of beta-adrenoreceptor blockade. Invest Ophthalmol Vis Sci 2011;52:155-70.

11. Chen J, Joyal JS, Hatton CJ, et al. Propranolol inhibition of $\beta$-adrenergic receptor does not suppress pathologic neovascularization in oxygen-induced retinopathy. Invest Ophthalmol Vis Sci 2012;53:2968-77.

12. Bancalari A, Schade R, Muñoz T, et al. Oral propranolol in early stages of retinopathy of prematurity. J Perinat Med 2016;44:499-503.

13. Korkmaz L, Baștuğ O, Ozdemir A, et al. The efficacy of propranolol in retinopathy of prematurity and its correlation with the platelet mass index. Curr Eye Res 2017;42:88-97.

14. Makhoul IR, Peleg O, Miller B, et al. Oral propranolol versus placebo for retinopathy of prematurity: a pilot, randomised, double-blind prospective study. Arch Dis Child 2013;98:565-7.

15. Filippi L, Cavallaro G, Bagnoli $P$, et al. Oral propranolol for retinopathy of prematurity: risks, safety concerns, and perspectives. J Pediatr 2013;163:1570-7.

16. Sanghvi KP, Kabra NS, Padhi $P$, et al. Prophylactic propranolol for prevention of ROP and visual outcome at 1 year (PreROP trial). Arch Dis Child Fetal Neonatal Ed 2017;102:F389-94.

17. Bührer C, Bassler D. Oral propranolol: a new treatment for infants with retinopathy of prematurity? Neonatology 2015;108:49-52.

18. Filippi L, Cavallaro G, Bagnoli $\mathrm{P}$, et al. Propranolol $0.1 \%$ eye microdrops in newborns with retinopathy of prematurity: a pilot clinical trial. Pediatr Res 2017;81:307-14.

19. Filippi L, Cavallaro G, Berti E, et al. Study protocol: safety and efficacy of propranolol $0.2 \%$ eye drops in newborns with a precocious stage of retinopathy of prematurity (DROP-ROP-0.2\%): a multicenter, open-label, single arm, phase II trial. BMC Pediatr 2017;17:165

20. International Committee for the Classification of Retinopathy of Prematurity. The international classification of retinopathy of prematurity revisited. Arch Ophthalmol 2005;123:991-9.

21. Bührer $C$, Erdeve Ö, van Kaam A, et al. N-terminal B-type natriuretic peptide urinary concentrations and retinopathy of prematurity. Pediatr Res 2017;82:958-63.

22. Faul F, Erdfelder E, Lang AG, et al. G*Power 3: a flexible statistical power analysis program for the social, behavioral, and biomedical sciences. Behav Res Methods 2007;39:175-91. 